\title{
Initial Experience of Transperineal Biopsy After Multiparametric Magnetic Resonance Imaging in Korea; Comparison With Transrectal Biopsy
}

\author{
Sung Goo Yoon ${ }^{1}$, Hyun Jung Jin', Jong Hyun Tae ${ }^{1}$, Tae II No ${ }^{1}$, Jae Yoon Kim ${ }^{1}$, \\ Jong Hyun Pyun ${ }^{2}$, Ji Sung Shim ${ }^{1}$, Sung Gu Kang', Jun Cheon', Jeong Gu Lee', \\ Je Jong Kim ${ }^{1}$, Deuk Jae Sung ${ }^{1,3}$, Kwan Hyi Lee ${ }^{4,5}$, Seok Ho Kang ${ }^{1}$ \\ ${ }^{1}$ Department of Urology, Korea University School of Medicine, Seoul, Korea \\ ${ }^{2}$ Department of Urology, Kangbuk Samsung Hospital, Sungkyunkwan University School of Medicine, Seoul, Korea \\ ${ }^{3}$ Department of Radiology, Korea University School of Medicine, Seoul, Korea \\ ${ }^{4}$ Department of Biomedical Engineering, University of Science and Technology, Seoul, Korea \\ ${ }^{5}$ Center for Biomaterials, Biomedical Research Institute, Korea Institute of Science and Technology, Seoul, Korea
}

\begin{abstract}
Purpose: The aim of this study is to confirm the detection rate of transperineal biopsy after multiparametric magnetic resonance imaging (mpMRI) and compared it to that of transrectal biopsy. We also examined the role of $\mathrm{mpMRI}$ and the rate of complications for each method.

Materials and Methods: In a retrospective study, we analyzed 147 patients who underwent mpMRI before prostate biopsy because of elevated serum prostate-specific antigen and/or abnormal digital rectal examination findings at Korea University Hospital, Seoul, Korea from March 2017 to April 2018. Regions on the mpMRI that were suggestive of prostate cancer were categorized according to the Prostate Imaging-Reporting and Data System (PI-RADS v2). For transperineal biopsy, a 20-core saturation biopsy was performed by MRI-TRUS cognitive or fusion techniques and a 12-core biopsy was performed in transrectal biopsy.

Results: Sixty-three and 84 patients were enrolled in transperineal group and transrectal group, respectively. The overall detection rate of prostate cancer in transperineal group was $27 \%$ higher than that in transrectal group. Classification according to PI-RADS score revealed a significant increase in detection rate in all patients, as the PI-RADS score increased. Frequency of complications using the Clavien-Dindo classifications revealed no significant differences in the total complications rate, but two patients in transrectal group received intensive care unit care due to urosepsis.

Conclusions: Our results confirmed that transperineal biopsy is superior to transrectal biopsy for the detection of prostate cancer. From the complication point of view, this study confirmed that there were fewer severe complications in transperineal biopsy. (Korean J Urol Oncol 2018;16:110-118)
\end{abstract}

Key Words: Prostate cancer - Transperineal prostate biopsy $\cdot$ MRI-TRUS fusion biopsy $\cdot$ PI-RADS score

Received September 10, 2018,

Accepted September 27, 2018

Corresponding Author: Seok Ho Kang

Department of Urology, Korea University Anam Hospital, Korea University School of Medicine, 73 Inchon-ro, Seongbuk-gu, Seoul 02841, Korea

E-mail: mdksh@korea.ac.kr

Tel: +82-2-920-6610, 5367, Fax: +82-2-928-7864

ORCID code: https://orcid.org/0000-0002-1524-5233

- This study has been supported by a research grant from the Korea University College of Medicine (Seoul, Korea).

\begin{abstract}
INTRODUCTION
Transrectal ultrasonography (TRUS)-guided prostate biopsy following an elevated (typically $>2.5-4.0 \mathrm{ng} / \mathrm{mL}$ ) serum prostate-specific antigen (PSA) level and/or a suspicious nodule on digital rectal examination (DRE) is currently the most widely
\end{abstract}

(7) (F) This is an Open Access article distributed under the terms of the Creative Commons Attribution Non-Commercial License (http://creativecommons.org/licenses/by-nc/4.0/) which permits unrestricted non-commercial use, distribution, and reproduction in any medium, provided the original work is properly cited. 2018 (C) Copyright The Korean Urological Oncology Society and The Korean Prostate Society. All Rights Reserved. 
used method for the diagnosis of localized prostate cancer. ${ }^{1-5}$ This method has several advantages, including requiring less time to perform, lower cost, requiring only readily available equipment, and being highly reproducible and easy to teach. ${ }^{6}$ The reason for these advantages is that the procedural time is mainly the time required to take the biopsies, and the procedure is performed under local anesthesia in a clinic room. However, fears have increased regarding undersampling of the prostate, particularly the anteroapical regions, ${ }^{4}$ and the growing rates of postbiopsy infections, leading to multidrug-resistant bacterial sepsis. ${ }^{5,7}$ Because of these problems, the interest in transperineal biopsy has increased in recent years. ${ }^{6}$ It is difficult to obtain an appropriate specimen from the anteroapical area by transrectal biopsy, but it is possible with transperineal biopsy. ${ }^{4}$ There is a relatively higher incidence of cancer in the anteroapical region than in other prostate areas, ${ }^{1,4}$ and transperineal biopsy, which can obtain adequate tissue in this area, can improve the detection rate. The transrectal biopsy needle passes through the patient's fecal material and may cause infection. However, such infections can almost completely be avoided in transperineal biopsy because there is no contact with the fecal material in this method. $^{7}$

With the increasing interest in transperineal biopsy, several studies have reported multiparametric magnetic resonance imaging (mpMRI) before biopsy to be helpful for accurate biopsy. ${ }^{3,8}$ mpMRI enables the surgeons to identify the location of prostate cancer lesions; in addition, its high negative predictive value (NPV) could solve the traditional problem of prostate biopsy, wherein cancer is not confirmed on histologic examination, but this problem cannot be excluded completely. Transperineal biopsy can obtain appropriate samples from the entire prostate, including the anteroapical area, compared with transrectal biopsy, and targeting of suspicious lesions is also advantageous. ${ }^{1}$ The combination of mpMRI and transperineal biopsy can lead to a synergistic effect.

To our knowledge, there were no study has assessed transperineal biopsy after mpMRI in Korea. In this study, we confirmed the detection rate of transperineal biopsy after mpMRI and compared it to that of transrectal biopsy performed at the same time. We also examined the rate of complications and the morbidity of each method.

\section{MATERIALS AND METHODS}

This study analyzed patients who underwent prostate biopsy at Korea University Hospital, Seoul, Korea from March 2017 to April 2018. We carried out mpMRI in patients with suspected prostate cancer, including those with PSA levels greater than $2.5 \mathrm{ng} / \mathrm{mL}$ (up to $20 \mathrm{ng} / \mathrm{mL}$ ) and/or abnormal DRE findings. Except for candidates unsuitable for MRI, all patients underwent mpMRI on a 3.0-T scanner (Siemens Medical System, Erlangen, Germany) with T1-weighted, biplanar T2weighted, and diffusion-weighted imaging, and the apparent diffusion coefficient was calculated.

Regions on the mpMRI that were suggestive of prostate cancer were categorized and indicated by three dedicated uro-radiologist according to the Prostate Imaging-Reporting and Data System, version 2 (PI-RADS v2), on a scale from 1 to 5, with higher scores indicating a greater likelihood of prostate cancer.

In patients who visited our hospital during this period, the biopsy method was determined by the physician's preference. Doctor Kang selected transperineal biopsy and other physicians in the same hospital selected transrectal biopsy. There were no other considerations in the selection of the biopsy method, and biopsy was performed with the same enroll criteria.

A total of 136 patients were enrolled in this study, including 57 who underwent transperineal biopsy (group A) and 79 who underwent transrectal biopsy (group B). Among these patients, 43 had a history of previous prostate biopsy, and there were 13 and 30 patients in groups A and B, respectively.

All biopsies were performed in the lithotomy position under general or local anesthesia in the operating room. To minimize complications related to anesthesia, monitored anesthesia care using propofol and fentanyl was performed and no muscle relaxants were used. In the case of transrectal biopsy, if patients had a high risk for general anesthesia, local anesthesia was administered.

Transrectal prostate sonography was performed using a biplanar transducer (BK Medical, Herlev, Denmark), and approximately 19-mm tissue samples were obtained using disposable 18-G needles (Bard Medical, Covington, GA, USA). For transperineal biopsy, a 20-core saturation biopsy (modified Barzelltemplate) was carried out by MRI-TRUS cognitive or fusion techniques, but fewer core biopsies were performed depending on the prostate size. In the case of transrectal biopsy, a 12-core 
biopsy was performed as described by Rodríguez-Covarrubias et al. ${ }^{9}$ We defined lesions with PI-RADS scores $\geq 3$ in mpMRI as 'regions of interest' and performed a maximum of six biopsies in these areas. A single urologist with multiple prostate biopsy experience performed the biopsies. Two dedicated uro-pathologists reviewed all the tissues.

According to the Clavian-Dindo system, complications were classified into 4 grades. Grade 1 complications required simple pharmacological treatments such as antiemetics, antipyretics, and analgesics. Complications requiring pharmacological treatment with drugs other than those administered for grade 1 complications were considered grade 2 complications, whereas grade 3 complications required surgical, endoscopic or radiological intervention. Life-threatening complications such as sepsis requiring intensive care unit (ICU) admission, were classified as grade 4 complications.

Statistical analyses were performed using IBM SPSS Statistics ver. 20.0 (IBM Co., Armonk, NY, USA).

\section{RESULTS}

Sixty-three and 84 patients were enrolled in groups A and $\mathrm{B}$, respectively. There was no significant difference in age, body mass index, PSA, abnormality of DRE, or PI-RADS scores between the 2 groups (Table 1). The prostate volume was significantly smaller in group A. The mean number of biopsy cores was about five more in group A. Comparison of operation time revealed that group A took about 29 minutes longer than group B.

The overall detection rate of prostate cancer in group A was $27 \%$ higher than that in group B and $27 \%$ higher in cases with prostate volumes above $50 \mathrm{~g}$ (Table 2 ). In patients with a previous prostate biopsy, the overall detection rate was $46.5 \%$; the rate in group A was $76.9 \%$, which was significantly higher than that in group B by $43 \%$ (Table 3 ).

Classification according to PI-RADS score revealed a significant increase in detection rate in all patients, group $\mathrm{A}$, and group $\mathrm{B}$ as the PI-RADS score increased (Table 4$)$. The detection rate of PI-RADS score $\geq 4$ in all patients was $73.5 \%$, and $86.8 \%$ in group A.

Analysis of the frequency of complications using the Clavien-Dindo classifications revealed no significant differences in the total complications rate between groups A and B (Table 5). Minor complications (grades 1 and 2) included postoperative hematuria, manual irrigation for blood clot removal, recatheterization due to urinary retention, and pain. There was no significant difference in minor complications between the two groups, but about $9 \%$ more complications were reported in

Table 1. Clinical characteristics of the study population

\begin{tabular}{|c|c|c|c|c|}
\hline Characteristic & Total $(n=147)$ & $\begin{array}{c}\text { Group A } \\
\text { (transperineal) }(n=63)\end{array}$ & $\begin{aligned} \text { Group } & B \\
\text { (transrectal) } & (n=84)\end{aligned}$ & p-value \\
\hline Age (yr) & $67.5 \pm 8.3$ & $67.2 \pm 8.5$ & $67.6 \pm 8.1$ & $0.770 *$ \\
\hline BMI $\left(\mathrm{kg} / \mathrm{m}^{2}\right)$ & $24.5 \pm 3.1$ & $25.0 \pm 3.2$ & $24.2 \pm 2.9$ & $0.108 *$ \\
\hline PSA (ng/mL) & $8.24 \pm 4.06$ & $8.43 \pm 4.27$ & $8.10 \pm 3.91$ & $0.619 *$ \\
\hline DRE nodule positive & & & & $0.843^{\dagger}$ \\
\hline Positive & $22(15.0)$ & $9(14.3)$ & $13(15.5)$ & \\
\hline Negative & $125(75.0)$ & $54(85.7)$ & $71(85.5)$ & \\
\hline Prostate volume $(\mathrm{mL})$ & $41.1 \pm 18.9$ & $37.0 \pm 15.1$ & $44.3 \pm 20.8$ & $0.021^{*}$ \\
\hline Biopsy core (n) & $15.8 \pm 4.0$ & $18.9 \pm 4.0$ & $13.4 \pm 1.7$ & $<0.001 *$ \\
\hline Procedure time (min) & $23.9 \pm 17.0$ & $40.0 \pm 13.3$ & $11.9 \pm 5.8$ & $<0.001 *$ \\
\hline PI-RADS score & & & & $0.201^{\dagger}$ \\
\hline$\leq 2$ & $22(15.0)$ & $6(9.5)$ & $16(19.0)$ & \\
\hline 3 & 42 (28.6) & $19(30.2)$ & $23(27.4)$ & \\
\hline 4 & $62(42.2)$ & $28(44.4)$ & $34(40.5)$ & \\
\hline 5 & $21(14.3)$ & $10(15.9)$ & $11(13.1)$ & \\
\hline
\end{tabular}

Values are presented as mean \pm standard deviation or number $(\%)$.

BMI: body mass index, PSA: prostate-specific antigen, DRE: digital rectal examination, PI-RADS: Prostate Imaging-Reporting and Data System.

${ }^{*}$ Independent t-test, ${ }^{\dagger}$ One-way analysis of variance test. 
Table 2. Comparisons of cancer detection rates and histopathologic characteristics

\begin{tabular}{|c|c|c|c|c|}
\hline Variable & Total & $\begin{array}{c}\text { Group A } \\
\text { (transperineal) }\end{array}$ & $\begin{array}{c}\text { Group B } \\
\text { (transrectal) }\end{array}$ & $\mathrm{p}$-value \\
\hline Group size (n) & 147 & 63 & 84 & \\
\hline $\mathrm{DR}$ of $\mathrm{PCa}$ & $82(55.8)$ & $45(71.4)$ & $37(44.0)$ & $0.001 *$ \\
\hline Group size $(\mathrm{PV}<50 \mathrm{~g})(\mathrm{n})$ & 103 & 48 & 55 & \\
\hline $\mathrm{DR}$ of $\mathrm{PCa}(\mathrm{PV}<50 \mathrm{~g})$ & $72(69.9)$ & $39(81.3)$ & $33(60.0)$ & 0.017 * \\
\hline Group size (PV $\geq 50 \mathrm{~g})(\mathrm{n})$ & 44 & 15 & 29 & \\
\hline $\mathrm{DR}$ of $\mathrm{PCa}(\mathrm{PV} \geq 50 \mathrm{~g})$ & $10(22.7)$ & $6(40.0)$ & $4(13.8)$ & 0.087 * \\
\hline Biopsy GS & 85 & 42 & 43 & \\
\hline GS 6 & $33(40.2)$ & $16(35.6)$ & $17(45.9)$ & $0.461^{\dagger}$ \\
\hline GS 7 & $14(17.1)$ & $10(22.2)$ & $4(10.8)$ & \\
\hline GS 8 & $32(39.0)$ & $16(35.6)$ & $16(46.2)$ & \\
\hline GS 9 & $3(3.5)$ & $3(7.1)$ & $0(0)$ & \\
\hline GS 10 & $0(0)$ & $0(0)$ & $0(0)$ & \\
\hline $\mathrm{GS} \geq 7$ & $49(57.6)$ & $29(69.0)$ & $20(46.5)$ & $0.001 *$ \\
\hline
\end{tabular}

Values are presented as number $(\%)$.

DR: detection rate, PCa: prostate cancer, PV: prostate volume, GS: Gleason score.

${ }^{*}$ Independent $\mathrm{t}$-test, ${ }^{\dagger}$ One-way analysis of variance test.

Table 3. Comparisons of cancer detection rates and histopathologic characteristics in patients with a history of biopsy

\begin{tabular}{|c|c|c|c|c|}
\hline Variable & Total & $\begin{array}{c}\text { Group A } \\
\text { (transperineal) }\end{array}$ & $\begin{array}{c}\text { Group B } \\
\text { (transrectal) }\end{array}$ & p-value \\
\hline Group size (n) & 43 & 13 & 30 & \\
\hline $\mathrm{DR}$ of $\mathrm{PCa}$ & $20(46.5)$ & $10(76.9)$ & $10(33.3)$ & $0.008 *$ \\
\hline Biopsy GS & & & & $0.013^{\dagger}$ \\
\hline Benign tissue & $23(53.5)$ & $3(23.1)$ & $20(66.7)$ & \\
\hline GS 6 & 5 (11.6) & $3(23.1)$ & $2(6.7)$ & \\
\hline GS 7 & $3(7.0)$ & $2(15.4)$ & $1(3.3)$ & \\
\hline GS 8 & $12(27.9)$ & $5(38.5)$ & 7 (23.3) & \\
\hline GS 9 & $0(0)$ & $0(0)$ & $0(0)$ & \\
\hline GS 10 & $0(0)$ & $0(0)$ & $0(0)$ & \\
\hline $\mathrm{GS} \geq 7$ & $15(34.9)$ & $7(53.9)$ & $8(26.6)$ & $0.013^{*}$ \\
\hline
\end{tabular}

Values are presented as number (\%).

DR: detection rate, PCa: prostate cancer, GS: Gleason score.

${ }^{*}$ Independent $\mathrm{t}$-test, ${ }^{\dagger}$ One-way analysis of variance test.

group A. Grade 3 complications requiring surgical, endoscopic, and radiological intervention did not occur in any patients. Two patients in group B received ICU care due to urosepsis, a grade 4 complication.

\section{DISCUSSION}

The results of this study confirmed the detection rate of transperineal biopsy after mpMRI for the first time in Korea. In a previously published systematic review of transperineal biopsy, the detection rate of the 12- and 24-core schemes were
$24 \%-75 \%$ and $75.9 \%$, respectively. ${ }^{1}$ The mean core number for transperineal biopsy in our study was about 18 , and the detection rate was $71.4 \%$, which was similar to that of previous studies. In addition, the detection rate of transperineal biopsy was higher than that of transrectal biopsy.

Although transperineal biopsy has the advantage of an improved detection rate, it is not widely used and there is still controversy about the choice of transperineal or transrectal biopsy as the diagnostic tool for prostate cancer. The reason is due to the many advantages of transrectal biopsy, including its implementation in a short period of time and its low-cost, sim- 


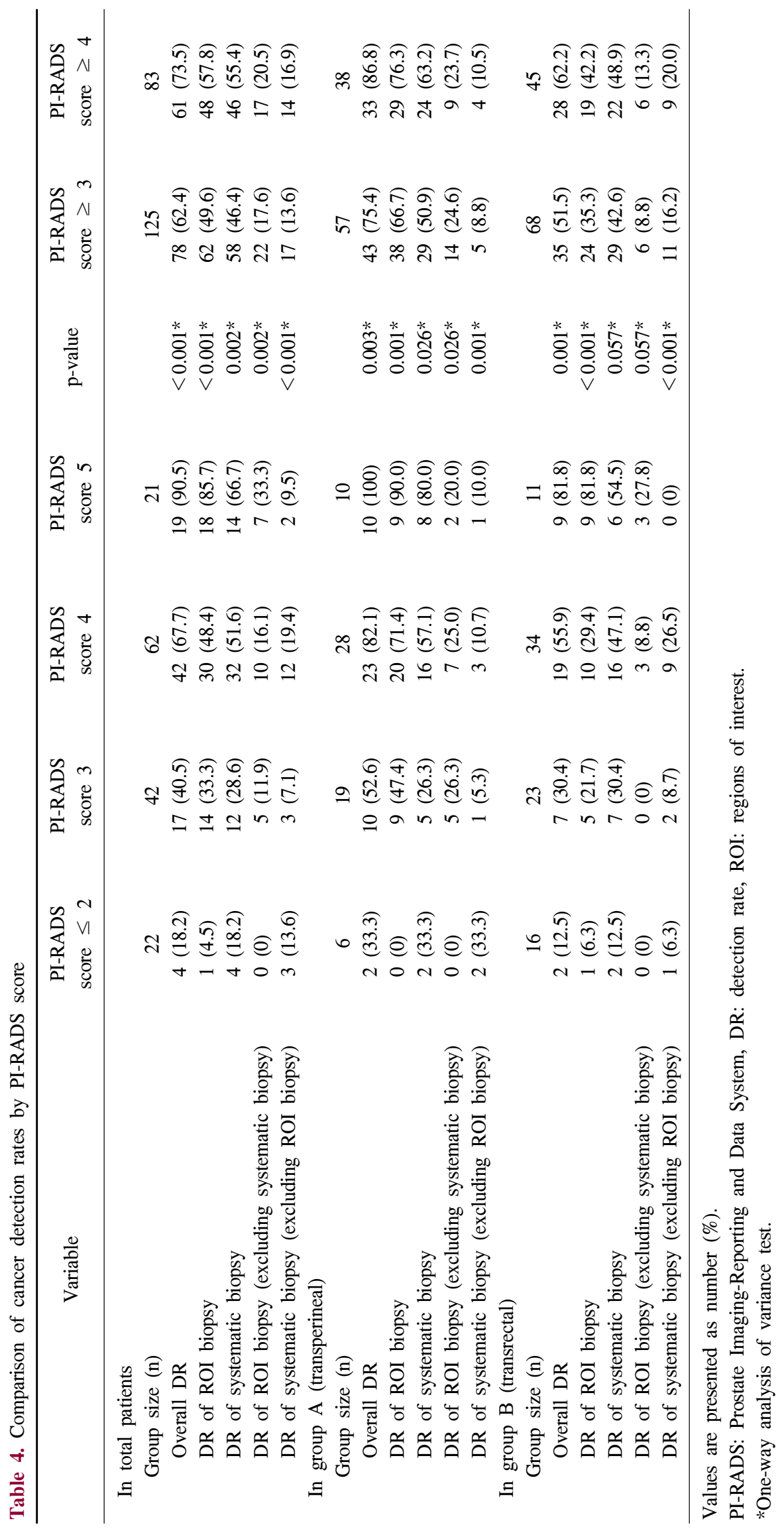


Table 5. Comparisons of complication rates

\begin{tabular}{lcccc}
\hline Grade & Total (n=147) & $\begin{array}{c}\text { Group A } \\
\text { (transperineal) }(\mathrm{n}=63)\end{array}$ & $\begin{array}{c}\text { Group B } \\
\text { (transrectal) }(\mathrm{n}=84)\end{array}$ & $\mathrm{p}$-value \\
\hline Positive & $55(37.4)$ & $27(42.9)$ & $28(33.3)$ & $0.244^{*}$ \\
Grade 1 & $39(26.5)$ & $17(27.0)$ & $22(26.2)$ & $0.915^{*}$ \\
Grade 2 & $14(9.5)$ & $10(15.9)$ & $4(4.8)$ & $0.023^{\dagger}$ \\
Grade 3 & $0(0)$ & $0(0)$ & $0(0)$ & - \\
Grade 4 & $2(1.4)$ & $0(0)$ & $2(2.4)$ & $0.220^{\dagger}$ \\
Grade 1 or 2 & $53(36.1)$ & $27(42.9)$ & $2(2.4)$ & $0.139^{*}$ \\
Grade 3 or 4 & $2(1.4)$ & $0(0)$ & & $0.220^{\dagger}$ \\
\hline
\end{tabular}

Values are presented as number (\%).

${ }^{*}$ Independent t-test, ${ }^{\dagger}$ Fisher exact test.

ple procedure. ${ }^{1}$ Transrectal biopsy requires only transrectal sonography and biopsy guns and can be performed under local anesthesia in the clinic room. It is also easy to learn and familiar to surgeons.

Despite the advantages of transrectal biopsy, interest in transperineal biopsy has increased in recent years. ${ }^{1}$ This is due to the superiority of sampling of transperineal biopsy in the anteroapical area and the increased incidence of quinolone-resistant Escherichia coli. ${ }^{1,7,10}$

It is difficult to obtain an appropriate specimen in the anteroapical area relatively far from the rectum by transrectal biopsy, but it is possible with transperineal biopsy. Many studies on the benefits of anteroapical sampling via transperineal biopsy and have emphasized its importance. ${ }^{4,11-13}$ Because of the relatively high incidence of cancer in the anteroapical area compared to that in other prostate areas, ${ }^{1}$ the false negative rate of transrectal biopsy may have been caused by this anatomical issue. In our study, $76.9 \%$ of the patients who had not been diagnosed with cancer on a previous prostate biopsy were diagnosed through transperineal biopsy. In cases of repeat transrectal biopsy, $33.3 \%$ of the patients were diagnosed with cancer, less than half the detection rate of transperineal biopsy.

Further, the recent increase in the incidence of fluoroquinolone-resistant E.coli is also problematic for transrectal biopsy. ${ }^{14}$ Because the biopsy needle passes through the rectal mucosa in transrectal biopsy, infection by the bowel flora of the rectum is possible. Many studies used povidone-iodine enema, preoperative rectal swab culture, and antibiotic change to reduce infection following transrectal biopsy, but consensus on the protocol has not yet been established, and it is still troublesome. ${ }^{5}$ In contrast, transperineal biopsy can be almost completely free from this infection risk because it passes through cleansed, sterilized skin rather than through the rectal mucosa. ${ }^{7}$ These superiorities of transperineal biopsy are valid reasons to replace transrectal biopsy.

The overall percentage of complications in transperineal biopsy analyzed by the Clavien-Dindo classification was slightly higher than that of transrectal biopsy, but the severity of complications was higher in transrectal biopsy. All transperineal biopsy complications were minor, including postoperative pain, hematuria, and recatheterization. Complications higher than grade 3 were not reported. In contrast, two patients in the transrectal biopsy group developed urosepsis and all received ICU care due to high fever and septic condition. The frequency of prostate biopsy complications varies widely. ${ }^{6}$ The most commonly reported complication is hematuria, which is reported in $10 \%-84 \%$ of cases, but most complications are self-limiting. Sepsis is reported in $0 \%-6.3 \%$ cases of transrectal biopsy, but no cases of sepsis on transperineal biopsy have been reported. ${ }^{10}$

In addition to the comparison of transperineal and transrectal biopsy, this study confirmed the benefit of mpMRI. Preoperative mpMRI is helpful for the localization and targeting of lesions suspicious of prostate cancer. In particular, the PI-RADS score of these images may help to objectify the reading and enable surgeons to predict the likelihood of prostate cancer. PI-RADS scores of 4 and 5 indicate that 'clinically significant cancer is likely or highly likely to be present' according to the American College of Radiology. In our study, PI-RADS score $\geq 4$ showed a $73.5 \%$ detection rate in all patients with both biopsy methods and a $90.5 \%$ rate for a score of 5 . In 288 biopsy-naive men, the detection rate of PI-RADS scores of 4 and 5 were $88 \%$ and $94 \%$, respectively, ${ }^{15}$ similar to those in the present study.

Furthermore, mpMRI showed a high NPV. The PROMIS 
study (2017) showed that the NPV of mpMRI for clinically significant lesions with a Gleason score (GS) of 7 was $76 \%$, whereas it ranged from $63 \%$ to $98 \%$ in previous studies. ${ }^{3}$ This high NPV might increase the number of men who could avoid prostate biopsy. ${ }^{3}$ If mpMRI were used as a triage test for prostate biopsy, one-quarter of men might safely avoid prostate biopsy. ${ }^{3}$ In contrast, the classical transrectal biopsy has a low NPV. Even if cancer was not detected on transrectal biopsy, prostate cancer cannot be completely ruled out. The false negative rate of transrectal biopsy was $47 \%$, and the rate of rebiopsy within five years after initial biopsy was $38 \% .{ }^{16}$ The high detection rate and NPV of mpMRI might provide benefits for screening patients who do not require biopsy.

Although there are concerns about the cost of implementing mpMRI before biopsy, mpMRI is cost-effective. The selection of patients with prebiopsy MRI can reduce unnecessary prostate biopsy. A study conducted in 2009 reported that the cost of unnecessary prostate biopsy exceeded the cost of prebiopsy mpMRI. ${ }^{17}$ In addition, a reduction in the total number of biopsy trials may reduce the social health cost spent by complications that occur after the biopsy, such as hematuria, rectal bleeding, and infection.

Moreover, prebiopsy mpMRI and its PI-RADS score may help to explain the need for biopsy and the diagnostic potential of cancer to patients. In planning the prostate biopsy, this modality will be a more objective indicator than conventional DRE and PSA, and it is expected to help improve the psychological stability of patients. ${ }^{17}$ In view of this advantage of mpMRI, preoperative imaging might be recommended as a routine procedure.

Until now, radical prostatectomy or radiation therapy have been performed for localized prostate cancer and active surveillance has been recommended in cases of low-risk prostate cancer. ${ }^{18,19}$ However, recent studies have questioned this method of treatment. First, radical prostatectomy for prostate cancer could be an overtreatment in some cases of clinically insignificant prostate cancer. ${ }^{20}$ The ProtecT study (2016) showed that clinically insignificant prostate cancer had a $6 \%$ disease progression over 10 years and less than $1 \%$ mortality during a follow-up of 6.8 years. $^{20}$ Insignificant cancer does not affect the mortality of the patient and does not progress to significant prostate cancer. ${ }^{21}$ In addition, there is the disadvantage of reducing the quality of life. ${ }^{19,20,22}$ Radical prostatectomy can lead to functional complications such as urinary incontinence and erectile dysfunction after the operation, which appear at a high frequency. In a study of 2,625 patients from 14 centers, Haglind et al. $^{23}$ found $21.3 \%$ and $70.4 \%$ incidence rates of incontinence and erectile dysfunction, respectively, at 12 months after robot-assisted laparoscopic prostatectomy. These complications are a major factor in reducing a patient's quality of life.

Second, radiotherapy has the potential to cause secondary malignancy and side effects due to the radiation of the surrounding organs. Wallis et $\mathrm{al}^{24}$ reported an increased risk of bladder, colorectal, and rectal cancer after radiotherapy. The Memorial Sloan Kettering Cancer Center group performed intensity-modulated radiation therapy (IMRT) or 3-dimensional conformal radiotherapy (3D-CRT) in 1,571 patients with T1-3 prostate cancer and followed-up the patients for 10 years. Although IMRT showed fewer complications than 3D-CRT, grade $\geq 2$ gastrointestinal and genitourinary complications were reported in $5 \%$ and $20 \%$ of cases, respectively. ${ }^{25}$ According to Wortel et al., ${ }^{26}$ following IMRT or 3D-CRT for the treatment prostate cancer, painful urination was reported in more than $50 \%$ of cases for both methods and grade $\geq 2$ genitourinary complications occurred in $38 \%$ and $48 \%$ of cases, respectively.

Third, active surveillance also contends with patient worry that they live with cancer, physician's anxiety that they may miss the appropriate time for treatment, and the risk of complications due to additional biopsy. In addition, there has been no clearly defined inclusion criteria for active surveillance and the consensus criteria are being renewed. ${ }^{27}$ The incidence of prostate cancer is increasing and the problems of these treatments are a strong trigger for increasing the social health burden.

Because of these problems, the direction of localized prostate cancer diagnosis and treatment is changing. With a more accurate and precise diagnosis, unnecessary diagnoses that are clinically insignificant can be avoided, thereby preventing overtreatment. ${ }^{3}$ Preoperative mpMRI and transperineal biopsy will help to improve this accuracy. As confirmed in our results, transperineal biopsy showed a higher diagnostic rate than that of transrectal biopsy and preoperative mpMRI enabled precise targeting for biopsy. The combination of transperineal biopsy and mpMRI will allow progress in the diagnosis of prostate cancer and will be indispensable for the future local treatment of prostate cancer. ${ }^{27}$

Transperineal biopsy and mpMRI are expected to expand the implementation of active surveillance. Accurate diagnosis through 
transperineal biopsy will help in screening patients for active surveillance, and mpMRI will be able to identify patients who require repeat biopsy to change the direction of treatment during active surveillance through mpMRI's high NPV. ${ }^{3}$ This would reduce patients' and physicians' anxiety and avoid repeat prostate biopsy that should be performed periodically. As radical treatments for prostate cancer do not show significant survival and metastatic benefits, more active surveillance will be performed with increased confidence about screening and follow-up testing.

In addition, focal therapy such as cryoablation and high-intensity focused ultrasound, which is a treatment method performed between radical prostatectomy and active surveillance, is expected to solve these functional and psychological problems. ${ }^{28}$ By treating partial prostate glands, focal therapy can avoid complications arising from radical prostatectomy and caused by radiation of radiotherapy and eliminate the anxiety associated with active surveillance. Accurate biopsy and diagnosis are essential for focal therapy, and confirmation of cancer location is required. This could be achieved through mpMRI and transperineal biopsy. Our study showed a synergistic effect for the combination of mpMRI and transperineal biopsy.

Our study has some limitations related to the study design. First, it was a retrospective study and we did not randomize patients and confirm the biopsy; thus, accurate detection rate comparisons may not be possible. However, although there was no randomization, our results showed good balance. Second, the small numbers of enrolled patients may show limited power to show a difference between transperineal and transrectal biopsy in our conclusion. Third, the difference between transperineal biopsy and transrectal biopsy was not corrected. As seen in our results, the core numbers of transperineal biopsy were higher than those of transrectal biopsy, and no compensation was made. Finally, we did not use contrast in the mpMRI protocol. This may have affected the PI-RADS scores because the dynamic contrast enhancement (DCE) in the score is a criterion to distinguish grades 3 and 4 in peripheral zone lesions. However, in terms of the cost and time, we believed that it would be beneficial not to use the contrast and PI-RADS scores of 3 and 4 were suspicious for prostate cancer. Although confirming the DCE could confirm a clearer PI-RADS score, it would not have made a significant difference in our research goals. Also, the role of DCE in the screening protocol is questionable, and Hausmann et al. ${ }^{29}$ recommended that DCE be used only for further evaluation of disease burden.

In summary, we have shown that transperineal biopsy increased the detection rate of prostate cancer and confirmed that there was a significant difference compared to the detection rate of transrectal biopsy. To our knowledge, these outcomes are the first results in Korea and they confirm that there are no differences in results compared to those of previous studies. Transperineal prostate biopsy with mpMRI may be a powerful tool for the diagnosis of prostate cancer. On the basis of this accurate pathologic examination, focal therapy can be performed to overcome the disadvantages of the existing treatment modalities. We believe that transperineal prostate biopsy with mpMRI may be used as a first recommended diagnostic method for prostate cancer.

\section{CONCLUSION}

Our results confirmed that transperineal biopsy is superior to transrectal biopsy for the detection of prostate cancer. From the complication point of view, this study confirmed that there were fewer severe complications in transperineal biopsy. The combination of mpMRI and transrectal biopsy will lead to changes in the diagnostic flow of prostate cancer.

\section{CONFLICT OF INTEREST}

The authors claim no conflicts of interest.

\section{REFERENCES}

1. Chang DT, Challacombe B, Lawrentschuk N. Transperineal biopsy of the prostate--is this the future? Nat Rev Urol 2013; 10:690-702.

2. Kasivisvanathan V, Rannikko AS, Borghi M, Panebianco V, Mynderse LA, Vaarala MH, et al. MRI-targeted or standard biopsy for prostate-cancer diagnosis. N Engl J Med 2018;378: 1767-77.

3. Ahmed HU, El-Shater Bosaily A, Brown LC, Gabe R, Kaplan R, Parmar MK, et al. Diagnostic accuracy of multi-parametric MRI and TRUS biopsy in prostate cancer (PROMIS): a paired validating confirmatory study. Lancet 2017;389:815-22.

4. Kawakami S, Kihara K, Fujii Y, Masuda H, Kobayashi T, Kageyama Y. Transrectal ultrasound-guided transperineal 14core systematic biopsy detects apico-anterior cancer foci of T1c prostate cancer. Int J Urol 2004;11:613-8.

5. Loeb S, Vellekoop A, Ahmed HU, Catto J, Emberton M, Nam R, et al. Systematic review of complications of prostate 
biopsy. Eur Urol 2013;64:876-92.

6. Huang H, Wang W, Lin T, Zhang Q, Zhao X, Lian H, et al. Comparison of the complications of traditional 12 cores transrectal prostate biopsy with image fusion guided transperineal prostate biopsy. BMC Urol 2016;16:68.

7. Acher P, Dooldeniya M. Prostate biopsy: will transperineal replace transrectal? BJU Int 2013;112:533-4.

8. de Rooij M, Hamoen EH, Fütterer JJ, Barentsz JO, Rovers MM. Accuracy of multiparametric MRI for prostate cancer detection: a meta-analysis. AJR Am J Roentgenol 2014;202: 343-51.

9. Rodríguez-Covarrubias F, González-Ramírez A, AguilarDavidov B, Castillejos-Molina R, Sotomayor M, Feria-Bernal G. Extended sampling at first biopsy improves cancer detection rate: results of a prospective, randomized trial comparing 12 versus 18 -core prostate biopsy. J Urol 2011;185:21326.

10. Grummet JP, Weerakoon M, Huang S, Lawrentschuk N, Frydenberg M, Moon DA, et al. Sepsis and 'superbugs': should we favour the transperineal over the transrectal approach for prostate biopsy? BJU Int 2014;114:384-8.

11. Takashima R, Egawa S, Kuwao S, Baba S. Anterior distribution of Stage T1c nonpalpable tumors in radical prostatectomy specimens. Urology 2002;59:692-7.

12. Satoh T, Matsumoto K, Fujita T, Tabata K, Okusa H, Tsuboi $\mathrm{T}$, et al. Cancer core distribution in patients diagnosed by extended transperineal prostate biopsy. Urology 2005;66:114-8.

13. Merrick GS, Gutman S, Andreini H, Taubenslag W, Lindert DL, Curtis R, et al. Prostate cancer distribution in patients diagnosed by transperineal template-guided saturation biopsy. Eur Urol 2007;52:715-23.

14. Feliciano J, Teper E, Ferrandino M, Macchia RJ, Blank W, Grunberger I, et al. The incidence of fluoroquinolone resistant infections after prostate biopsy--are fluoroquinolones still effective prophylaxis? J Urol 2008;179:952-5; discussion 955.

15. Washino $\mathrm{S}$, Okochi $\mathrm{T}$, Saito $\mathrm{K}$, Konishi $\mathrm{T}$, Hirai $\mathrm{M}$, Kobayashi $\mathrm{Y}$, et al. Combination of prostate imaging reporting and data system (PI-RADS) score and prostate-specific antigen (PSA) density predicts biopsy outcome in prostate biopsy naïve patients. BJU Int 2017;119:225-33.

16. Sonn GA, Chang E, Natarajan S, Margolis DJ, Macairan M, Lieu $\mathrm{P}$, et al. Value of targeted prostate biopsy using magnetic resonance-ultrasound fusion in men with prior negative biopsy and elevated prostate-specific antigen. Eur Urol 2014;65:80915 .

17. Ahmed HU, Kirkham A, Arya M, Illing R, Freeman A, Allen $\mathrm{C}$, et al. Is it time to consider a role for MRI before prostate biopsy? Nat Rev Clin Oncol 2009;6:197-206.

18. Graham J, Kirkbride P, Cann K, Hasler E, Prettyjohns M. Prostate cancer: summary of updated NICE guidance. BMJ 2014;348:f7524.

19. Ghai S, Trachtenberg J. MRI-guided biopsies and minimally invasive therapy for prostate cancer. Indian J Urol 2015;31: 209-16.

20. Hamdy FC, Donovan JL, Lane JA, Mason M, Metcalfe C, Holding $\mathrm{P}$, et al. 10-Year outcomes after monitoring, surgery, or radiotherapy for localized prostate cancer. N Engl J Med 2016;375:1415-24.

21. Ploussard G, Epstein JI, Montironi R, Carroll PR, Wirth M, Grimm MO, et al. The contemporary concept of significant versus insignificant prostate cancer. Eur Urol 2011;60:291303.

22. Montorsi F, Wilson TG, Rosen RC, Ahlering TE, Artibani W, Carroll PR, et al. Best practices in robot-assisted radical prostatectomy: recommendations of the Pasadena Consensus Panel. Eur Urol 2012;62:368-81.

23. Haglind E, Carlsson S, Stranne J, Wallerstedt A, Wilderäng $\mathrm{U}$, Thorsteinsdottir $\mathrm{T}$, et al. Urinary incontinence and erectile dysfunction after robotic versus open radical prostatectomy: a prospective, controlled, nonrandomised trial. Eur Urol 2015; 68:216-25.

24. Wallis CJ, Mahar AL, Choo R, Herschorn S, Kodama RT, Shah PS, et al. Second malignancies after radiotherapy for prostate cancer: systematic review and meta-analysis. BMJ 2016;352:i851.

25. Zelefsky MJ, Levin EJ, Hunt M, Yamada Y, Shippy AM, Jackson A, et al. Incidence of late rectal and urinary toxicities after three-dimensional conformal radiotherapy and intensitymodulated radiotherapy for localized prostate cancer. Int $\mathbf{J}$ Radiat Oncol Biol Phys 2008;70:1124-9.

26. Wortel RC, Incrocci L, Pos FJ, Lebesque JV, Witte MG, van der Heide UA, et al. Acute toxicity after image-guided intensity modulated radiation therapy compared to $3 \mathrm{D}$ conformal radiation therapy in prostate cancer patients. Int $\mathrm{J}$ Radiat Oncol Biol Phys 2015;91:737-44.

27. Bokhorst LP, Valdagni R, Rannikko A, Kakehi Y, Pickles T, Bangma $\mathrm{CH}$, et al. A Decade of active surveillance in the PRIAS study: an update and evaluation of the criteria used to recommend a switch to active treatment. Eur Urol 2016;70: 954-60.

28. Lindner U, Trachtenberg J. Focal therapy for localized prostate cancer -choosing the middle ground. Can Urol Assoc J 2009;3:333-5.

29. Hausmann D, Aksöz N, von Hardenberg J, Martini T, Westhoff N, Buettner S, et al. Prostate cancer detection among readers with different degree of experience using ultra-high b-value diffusion-weighted Imaging: is a non-contrast protocol sufficient to detect significant cancer? Eur Radiol 2018;28:869-76 